\title{
Hand Immobilization Causes Changes In Cortical Areas: qEEG Alpha Band Absolute Power Study
}

Dionis Machado1,2, Victor Hugo do Vale Bastos², Silmar Teixeira1,2, Maurício Cagy², Ada Sandoval-Carrillo4, Jose Salas-Pacheco ${ }^{4}$, Sergio Machado7,8,9,10, Bruna Velasques 1,5,6, Pedro Ribeiro1,5,6, Oscar Arias-Carrión11,12,*

\section{Abstract}

Hand immobilization has been associated with changes in neural networks of primary somatosensory cortex and primary motor areas. Electrophysiologically, alpha band absolute power may indicate how cerebral cortex processes information. This study aimed to analyze changes in alpha band absolute power on frontal, central, parietal and occipital derivations when hand-movement of subjects was restricted for 48 hours. Fifteen healthy volunteers (20 to 30 years old), were recorded using electroencephalography (qEEG), while exposition to visual stimulus linked to a motor task before and after hand immobilization. Statistical analysis revealed that hand immobilization caused changes in frontal, central and parietal areas of cerebral cortex. In summary, after hand immobilization alpha band absolute power increased in these areas, revealing a lower activation. Contrarily, at C4 there was a decreased alpha band absolute power correlated to more activation. These findings can be due adaptive plasticity to supply less activation at $\mathrm{C} 3$, considering the inactivity of right hand due to the immobilization. Further studies are needed to better understand the complex processes involved in this type of task.

\section{Keywords}

Alpha band, qEEG, immobilization, neural plasticity

\section{Background}

Cerebral cortex is a dynamic structure which changes in its organization due a specific condition. This fact can be observed in situations like motor learning and rehabilitation [1]. Particularly, cerebral cortex shows reorganization after a lesion when movements be-
1 Brain Mapping and Sensory Motor Integration, Institute of Psychiatry of Federal University of Rio de Janeiro (IPUB/UFRJ), Brazil.

2 Brain Mapping and Functionality Laboratory (LAMCEF/UFPI), Federal University of Piauí, Brazil.

3 Bioengenieren Program, COPPE, Federal University of Rio de Janeiro, Rio de Janeiro, Brazil.

4 Instituto de Investigación Científica, Universidad Juárez del Estado de Durango. Durango, Durango, México.

5 School of Physical Education, Bioscience Department (EEFD/UFRJ), Brazil

6 Institute of Applied Neuroscience (INA), Rio de Janeiro, Brazil.

7 Panic and Respiration, Institute of Psychiatry of Federal University of Rio de Janeiro, Brazil.

8 National Institute for Translational Medicine (INCT-TM), Brazil.

9 Quiropraxia Program of the Faculty of Health Sciences, Central University, Santiago, Chile.

10 Physical Activity Neuroscience, Physical Activity Sciences Postgraduate Program Salgado de Oliveira University, Niterói, Brazil.

11 Unidad de Trastornos del Movimiento y Sueño (TMS), Hospital General Dr. Manuel Gea González/IFC-UNAM, México D.F., México.

12 Unidad de Trastornos del Movimiento y Sueño (TMS), Hospital General Ajusco Medio, México D.F., México.

Contact information:

” arias@ciencias.unam.mx 
come temporarily or permanently impaired such as in accident that involves immobilization or in a stroke which some cortical functions are affected $[2,3]$. Understanding these alterations can be useful to propose rehabilitation modalities to restore movements. In this way, hand movements appear to be an interesting source of study due to motor and sensorial representations in the cerebral cortex [4]. Moreover, studies found changes in neural networks in primary somatosensory cortex and primary motor areas (M1) when individuals were subjected limited movements $[2,5,6]$.

Studies had investigated cortical changes occurring after hand immobilization using electroencephalography (qEEG) which consists on useful tool to detect cortical changes associated to especial conditions, such immobilization or using a rehabilitation modality to improve motor function $[3,5]$. Generally qEEG have been employed to observe changes in cortical activity due tasks or use of some drugs $[7,8]$. Furthermore, alpha band absolute power may indicate how cerebral cortex processes information, with more mental effort or with less one. Alpha band $(8-12 \mathrm{~Hz})$ has shown to be strongly associated with attention, cognition, perception and motor process, being related to alertness and cognitive tasks involving attention [9-12]. We are interested on alpha band due to relationship with a relaxation state and with slowness in psycho-physiological process [13]. In addition, the frontal areas are engaged in motivation mechanism, planning to execute voluntary movements, whereas parietais areas are involved in sensorial control mechanism [14-17]. Thus, study involving analysis of the frontal and parietal regions may indicate their functional response in task which requiring specific attention.

Previous studies have been conducted to show the involvement of the cerebral cortex reorganization after motor function limitation condition [5, 18]. But does how cerebral cortex processes visual information linked to a motor task after a condi- tion of immobilization? For this purpose, we seek to answer the following questions: i) Does hand immobilization cause limitation of sensory afferences? ii) Does cerebral cortex changes its activation after 48 hour of the hand immobilization in order to attend the task? Moreover, the activation of occipital areas may be not affected by immobilization, but the activation of sensory motor integration areas may be influenced by absence of movement resulting of immobilization. Another hypothesis is that a competition between stimuli can occurs, in other words, a decreased of attention may happen to the visual stimulus when motor task be required. Thus, our study objectived to analyze the neurophysiological changes occurring in cerebral cortex during visual stimulus exposition linked to a motor task before and after of the hand immobilization. Specifically, this study aimed to analyze changes on alpha band absolute power in the frontal, central, parietal and occipital regions when individuals were subjected at 48 hours of hand immobilization.

\section{Methods}

\section{Sample}

The sample was composed of 15 healthy individuals: 04 men and 11 women, with ages varying between 20 and 30 (mean age $24 \pm 1.2$ years). The individuals were chosen randomly and the recruitment of the volunteers was accomplished thanks to the research announcements posted in different Universities of Rio de Janeiro State. As inclusion criteria, the subjects needed to be right handed, have no mental or physical illness (previous anamnese) and not use any psychoactive or psychotropic substances during the whole time of the study. A detailed questionnaire was applied in order to exclude those individuals who could contaminate the results. Due to hand laterality, the Edinburgh inventory $[19,20]$ was utilized to identi- 
fy the predominance of the participants (right handed vs left-handed). Consequently, the left-handed individuals were excluded from the experiment. The individuals were instructed to not use tobacco, coffee or alcoholic drinks 10 hours before the test because these substances can influence cortical activation recorded by qEEG [21-23]. The participants received written information about the study procedures and we solicited their signature of the consent form. This study was approved by the ethics committee of Veiga de Almeida University with the number 149,817 in accordance with the ethical standards laid down in the 1964 Declaration of Helsinki.

\section{Tasks and Procedures}

The experimental procedure was conducted on a room with acoustic and electrical isolation. During the electroencephalography (EEG) signal acquisition, the lights were dimmed. The subjects sat in a chair with armrest in order to minimize muscle artifact during EEG signal acquisition. In front of the subjects there was a 15-inch monitor on a table. The monitor was turned on only when the subjects executed the task (i.e, flexion and extension of the index finger). Initially, the EEG signal acquisition lasted for 2 minutes (rest) with the monitor off facing the subjects. Then, a sensor to measure acceleration (accelerometer) was coupled on the right index finger; during the visual feedback, the subjects executed the task (i.e, flexion and extension index finger). The accelerometer was connected to the EEG with an additional channel (i.e, channel 21). When the subjects performed the movement, the accelerometer provided a signal for the EEG.

The subjects were instructed to perform the index finger flexion and extension when visual feedback was generated by a random image on the monitor. The subjects executed the task in 6 blocks of 15 trials. In order to avoid muscle fatigue, they rested 3 minutes between each block. Thus, the task has 1 minute each block with 3 minutes interval between blocks which 24 minutes totals task. After completing the task, the monitor was turned off and the subjects were submitted again to EEG during $2 \mathrm{mi}-$ nutes (rest). After EEG recording, a plaster cast was applied on the subjects' right hand and they kept it on for 48 hours. The plaster cast was applied with hand closed in order to preventing any hand or finger movement. After this period, subjects returned to the laboratory to remove the plaster cast and they were after five minutes plaster cast removed again submitted to the task procedures before hand immobilization.

\section{Data acquisition \\ Electroencephalography}

The International 10/20 system for electrodes was used with 20-channel Braintech-3000 EEG system (EMSA-Medical Instruments, Brazil). The 20 electrodes were arranged in a nylon cap (ElectroCap Inc, Fairfax, VA, USA), yielding mono-pole derivations to linked earlobes. In addition, two 9-mm diameter electrodes were attached above and on the external corner of the right eye, in a bipolar electrode montage, to monitor artifacts on eye-movements (EOG). Impedance of EEG and EOG electrodes was kept under 5-10 $\mathrm{K} \Omega$. The data acquired had total amplitude of less than $100 \mu \mathrm{V}$. The EEG signal was amplified with a gain of 22.000, analogically filtered between $0.01 \mathrm{~Hz}$ (high-pass) and $100 \mathrm{~Hz}$ (lowpass), and sampled at $240 \mathrm{~Hz}$. The software Data Acquisition (Delphi 5.0) was employed to filter the raw data: notch $(60 \mathrm{~Hz})$, high-pass of $0.3 \mathrm{~Hz}$ and low-pass of $100 \mathrm{~Hz}$.

\section{Data processing}

To quantify reference-free data, a visual inspection and independent component analysis (ICA) was applied to identify and remove any remaining artifacts, i.e, eye blinks and ocular movements, produced by the task [24]. Data from individual electrodes exhibiting loss of contact with the scalp or high impedances $(>10 \mathrm{k} \Omega$ ) were discarded, and 
data from single-trial epochs exhibiting excessive movement artifacts $( \pm 100 \mu \mathrm{V})$ were also deleted. ICA was then applied to identify and remove any artifacts after the initial visual inspection. ICA is an information maximization algorithm to blind the EEG signals related to the artifacts [24-26]. Independent components resembling eye-blink or muscle artifacts were removed and the remaining components were then projected back onto the electrode data by multiplying it by the inverse matrix of the spatial filter coefficients derived from ICA, using established procedures. The ICA-filtered data were then reinspected for residual artifacts using the same rejection criteria described above. Then, a classic estimator was applied for the power spectral density, or directly from the square modulus of the Fourier Transform, performed by MATLAB (Matworks, Inc.). Quantitative EEG parameters were reduced to $4 \mathrm{~s}$ periods (the selected epoch started $2 \mathrm{~s}$ before and ended $2 \mathrm{~s}$ after visual stimulus).

\section{Selected derivations and frequency band}

Derivations in thefrontal region (Fp1, Fp2, F3, F4, F7, F8 and Fz) were selected due to their functional relationship with motivation, planning and motor program [26]. Other derivations were selected due to their relationship with motor act (C3, Cz and C4) [27]. The derivations representing parietal region (P3, Pz and P4) were chosen due to their functional relationship with sensorimotor integration [28]. Moreover we selected derivations in the occipital region ( $\mathrm{O} 1, \mathrm{Oz}$ and $\mathrm{O} 2$ ) were chosen because its association with visual function that was necessary to respond the stimulus. Alpha band was chosen because shows relationship with relaxation state or slowness of psychophysiological processes and can indicates mental effort of cerebral cortex in processes information. Furthermore, alpha band have been associated with attentional suppress per visual and somatosensory systems [29-32]. Beside, absolute power was elected because corresponds to total energy of a derivation on a certain region at different frequency bands [33].

\section{Statistical Analysis}

The statistical design allowed the examination of functional cortical action before and after 48 hour of hand immobilization. Areas in each region, with respective regions related to sensory, motor execution, and integrative or associative functions (i.e, electrodes Fp1, Fp2, F3, F4, F7, F8, Fz, C3, C4, Cz, P3, P4, Pz, 01, O2, Oz) were selected. Data were standardized and normalized into values of absolute power using homocedasticity in a natural logarithmic test (LogN) in order to approximate values to a normal distribution [34-35]. Thus, two factorial designs were employed for alpha band with factor moment (before vs after visual stimuli) and factor conditions (before vs after 48 hours hand immobilization). The interaction was investigated with one-way ANOVA with factor condition for each EEG acquisition. The significance levels were set at $p \leq$ .005 . These analyses were conducted utilizing the SPSS for Windows version 18.0 (SPSS Inc, Chicago, II, USA).

\section{Results and discussion}

The results of two-way ANOVA showed main effect for condition (i.e, before immobilization vs after immobilization) at Fp2 derivation $[F(1,3812)=4.032$; $p=0.045]$. The statistical analysis showed an increased on alpha band absolute power after hand immobilization, leading to believe that immobilization influenced alpha oscillation. A one-way ANOVA was used in order to investigate interaction. Thus, the qEEG analysis at Fp1 derivation showed interaction for condition (i.e, before immobilization vs after immobilization) and moment (i.e, before visual stimulus vs after visual stimulus) $[F(1,3841)=5.430$; $p=0.02$ ] (Figure 2). Our statistical results of oneway ANOVA for interaction was $[F(3,3844)=7.924$; $p=0.001]$. Post hoc Bonferroni test showed signifi- 
Figure 1: A) An interaction for condition (before immobilization in first visit versus after immobilization in second visit) and moment (before versus after visual stimulus) in left fronto polar cortex (Fp1) ( $p<.001)$. B) Mean and SD indicate main effect for condition (before immobilization in first visit versus after immobilization in second visit) observed in right fronto polar cortex (Fp2) ( $p=.045)$.

A

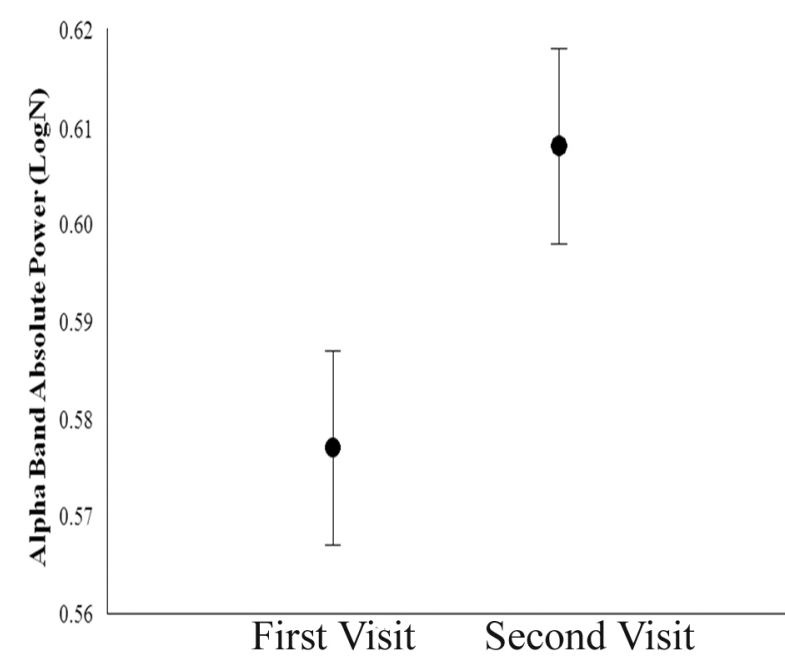

B

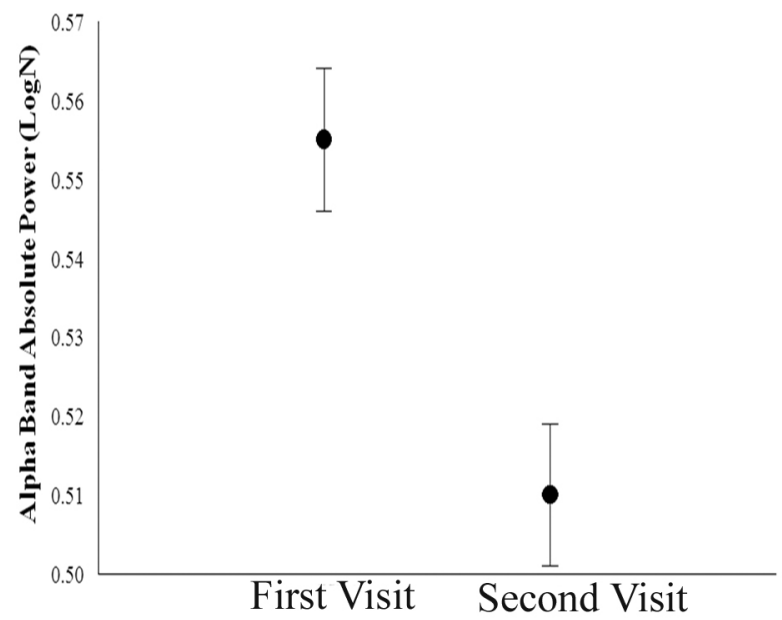

Figure 2: a) Main effects for factor treatment observed in the left central cortex (C3) derivation by mean and SD $(p<.001)$;

b) Main effects for factor treatment observed in the left parietal cortex (P3) derivation by mean and SD $(p<.001)$.

\section{A Left Anterior Prefrontal Cortex}

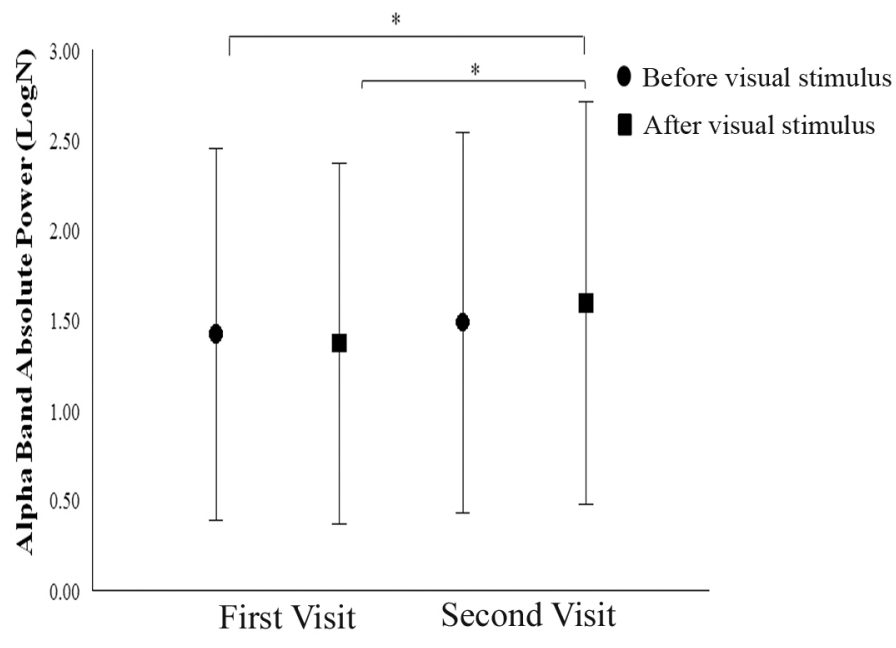

\section{B Right Anterior Prefrontal Cortex}

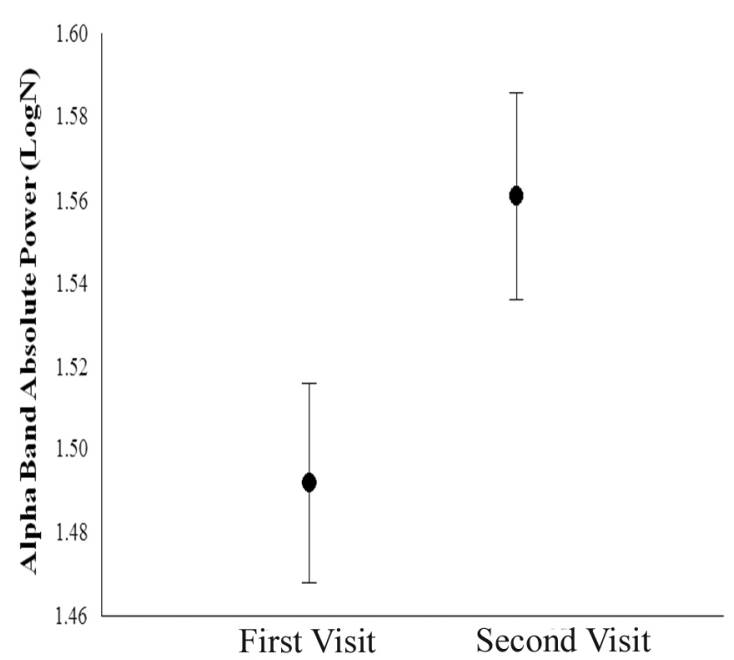

cant difference between pre and post hand immobilization. There was an increased on alpha band absolute power comparing pre immobilization be- fore stimulus (mean $1.42 \pm 1.03$ ) with post hand immobilization after stimulus (mean $1.59 \pm 1.11$ ). Similarly, an increased on alpha band absolute power 
occurred when compared pre immobilization after stimulus (mean $1.37 \pm 0.99$ ) with post hand immobilization after stimulus (mean $1.59 \pm 1.11$ ). Probably the immobilization influenced the activation on frontal polar areas (Figures 1A and 1B).

In central region, the two-way ANOVA showed main effect at $[C 3 F(1,3585)=4.642 ; p=0.031]$ (Figure 2A) and [C4 $F(1,3647)=12.103 ; p=0.001]$ (Figure 2B) for condition (before vs after immobilization). An increased on alpha band absolute power was seen at C 3 derivation between before (mean $0.577 \pm 0.010$ ) and after (mean $0.608 \pm 0.010$ ) hand immobilization. The opposite occurred at C4 derivation which showed before (mean $0.555 \pm$ 0.009 ) and after (mean $0.510 \pm 0.009$ ) hand immobilization a decreased on alpha band absolute power. These findings demonstrated that C3 and C4 derivations act differently when individual are submitted the hand immobilization. Moreover, C4 derivation may went more activate because 48 hours of the hand immobilization, subjects used more the left hand due immobilization of the right one.

The two-way ANOVA implemented to analyses parietal region revealed significative difference at P4 derivation, $[F(1,3296)=5.072 ; p=0.024]$ (Figure 3). An increased on alpha band absolute power occurred after hand immobilization. In contrast to the results found in frontal, central and parietal areas, there were no significative differences on occipital region, $O 1(p=0.51), O 2(p=0.83)$ and $O z(p=0.70)$ showing that this region was not influenced by hand immobilization according us expected because the immobilization may be more associate between sensory and motor than visual function.

This study was conducted in order to analyze the neurophysiological changes occurring in cerebral cortex during an exposition to visual stimulus linked to a motor task before and after an immobilization condition of the hand. Thereby, the study aimed to analyze changes in alpha band absolute power on frontal, parietal and occipital derivations when
Figure 3: Main effect for condition (before immobilization in first visit versus after immobilization in second visit) in the right parietal cortex (P4) derivation by mean and SD $(p=.024)$.

\section{Right Parietal Cortex}

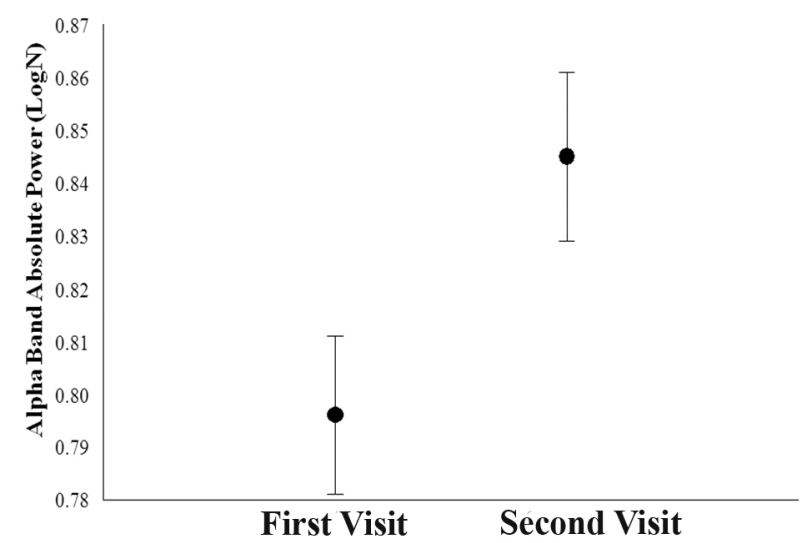

individuals are subjected to restriction of movement for 48 hours. Moreover, was hypothesized that after 48 hours of hand immobilization cortical changes occur in the ipsi- and contralateral hemisphere, was expected find changes in absolute power of the derivations studied, because it represents a fraction of the signal power concentrated in alpha band.

We observed that alpha band absolute power increased at Fp2 derivation after hand immobilization. Thus, the frontal region; in special, the frontopolar cortex seems be activated during task which involving planning, problem solving and reasoning [36]. Studies indicate that frontopolar cortex has particular importance in cognitive function, highlighting its role in to hold goals in mind while exploring and processing secondary goals, process named multitasking [37-40]. Study with functional magnetic resonance imaging revealed that bilateral regions in the frontopolar prefrontal cortex are selectively activated when subject hold in mind goals while simultaneously process secondary goals (dual-task), this process usually required in planning and reasoning [37]. 
The task proposed in our experimental design can be understood like dual-task because required processing visual information and performance the motor task (i.e, flexion and extension index finger). The increased on alpha band absolute power at Fp2 derivation after hand immobilization may indicated lapse in attention to the performance of dual-task [41], and may reflect the fact that the task does not require functions linked to the frontopolar region, i.e. planning, problem solving and reasoning [36]. The activity increased on the alpha band usually happen in subjects who has attention deficit hyperactivity disorder [12], but it is worth noting that volunteers of this study were assessed by previous anamneses which objective consisted in exclude those who had mental illness. Moreover, Fp2 derivation is linked to the left corporal side that was neither submitted to the immobilization nor involved in motor task. It may be consider that volunteers of this study were right handed, so a possible change occured at Fp1derivation due to its relationship with right corporal side that was involved by hand immobilization and recruited in motor task.

The interaction found at Fp1 derivation for condition (before vs after immobilization) and moment (before vs after visual stimulus) evidenced an increased on alpha band absolute power, i.e. alpha band absolute power was higher after visual stimulus and after immobilization than when compared to before visual stimulus and before hand immobilization (Figure 2). Probably, before immobilization and visual stimulus the neural circuitry was in readiness to identify visual stimulus and execute motor task. Thus, we understood that after hand immobilization and visual stimulus, the increased on alpha band absolute power indicates that this circuitry became less activated [42]. The immobilization may have caused less afference to right hand and absence of movement made the region less active. A research involving work memory indicated prefrontal areas involvement in visual-spatial tasks that required considerable level of difficulty, and when this occurred, there was less activation in alpha band [43]. The involvement of prefrontal areas in executive functions is reflected by a decrease of alpha. In our study, the finding of high values in alpha band in frontal areas may denote a feature of the task that not required executive functions (easiness task).

Furthermore, visual stimulus was presented for 15 times in each block, totalizing 90 stimuli per six blocks. Like this, after several presentations of the visual stimulus, the attention level decreased because the subjects knew what would be presented to them, especially in condition after immobilization because they had knowledge about the experimental procedure. The increased on alpha band absolute power reflect habituation mechanism, which is viewed in some studies like a process in which the response to a repetitive stimuli tends to decrease [44-45]. Besides, researches indicate that combined tasks leading to suppression of neural circuitry activity accountable for

execution of the first task while areas involved in the second one showing consequently increase in its activation [46-47]. Perhaps, in our study, the performance to the motor task (flexion and extension of the index finger) suppressed the neural circuitry activity at region of Fp1 derivation during the identification of the visual stimulus according Foxe \& Simpson (2005) that using high-density mapping of human event-related potentials examined the brain activity associated with selective information processing when subjects were cued on a trial-by-trial basis to perform a discrimination in either the visual or auditory modality. These authors suggest that when individuals are required to switch rapidly from execution of one task to another, goal-related task networks and attentional mechanisms are engaged to reconfigure task-specific networks, suppressing activity within circuits responsible for performance of the old task and amplifying preparatory neural processes for the anticipated novel task.

We observed increased at C3 derivation after hand immobilization probably, due to the relation- 
ship between right hand with $\mathrm{C} 3$ derivation which is involved with motor act [48]. Thereby, the right hand immobilization may diminished afference resulting in less activation in the central area which showed high values in alpha band after hand immobilization. Indeed, ours findings are according studies which indicate that occur plastic changes on motor cortex at the area of hand's representation when occurr hand immobilization [3]. Several studies indicate an adaptive plasticity per less representation of the cortical area after amputation or movement restriction [49-51]. The literature shows that chronic reduction on neuromuscular activity caused by immobilization results in muscular atrophy, weakness and damage to the motor function linked to changes on cortical excitability [49]. A sensorimotor restriction causes damage to motor function due structurally and functionally reorganization of sensorimotor cortex [3]. Study involving microscopy verified that 14 days of immobilization of hind feet of rats are sufficient to cause decrease on dendritic spine length and others morphological changes associated to reorganization of motor cortex in response to the decrease on sensorimotor function [50]. In this sense, we highlight that immobilization can alter neural networks responsible to the motor act.

Corroborating our findings, a study involving saccadic task also found increase on alpha band absolute power at C3 after visual stimulus. The authors correlate this finding to the automation of the task; in others words, high levels of attention were less necessary to execute the task [52]. In our study, we understood that before hand immobilization functioned as training. In this case, at condition after hand immobilization the subject knew the task which promoted less attention lever for its execution. Furthemore, the task repetition caused less recruitment in the central area, which may signalize learning of the task [52].

Contrary to the others derivations, at C4 alpha band absolute power decreased, leading to believe that this region was more activated. Thus, the inactivity of right hand due to hand immobilization caused adaptive plasticity at C3 and demanded highest mental effort of corresponding contralateral area to attend the task [42]. Studies have shown that intact neurons can compensate function loss of neurons injured. Thereby, hand immobilization had created diminish of afference reflecting in less activity at C3 area which to be compensate required participation of the contralateral homologous cortical area (C4) [53-54]. This compensation seems occur via corpus callosum which has been observed on studies involving patients, healthy subjects and even chimpanzee [55-56].

Studies involving functional magnetic resonance imaging revealed that amputation and deafferentation induce changes on sensorial (S1) and motor (M1) cortex [57-59]. Likewise, Viaro et al. (2014) [51], using intracortical microstimulation in adults rats, found cortical changes due to movement restriction. Traditionally, it was knew that cortical area responsible per sensorimotor behavior of amputated segment was transferred to functionalities of neighboring regions [60]. Simões et al. (2012) [57]; related a reconfiguration beyond this one, they found changes on cortical representation of the intact segment (not amputated). For example, in amputated subjects, tactile stimulation on intact foot induced high activation on S1 and M1 ipsilateral when compared with subjects no amputated (control). This finding according to our findings of highest cortical activation ipsilateral to the hand immobilized. The hand immobilization per 48 hours can function as a deafferentation mechanism [51].

In parietal region was seen an increased on alpha band absolute power at derivation P4. Anatomically, the control of spatial attention is localized at dorsal frontoparietal regions [61]. Recent evidences denote an important role of the posterior parietal cortex on attention-related modulation of visual cortex activity [62]. Study used repetitive Transcranial Magnetic Stimulation observes that it involved 
interference of intraparietal cortex during allocation of spatial attention which causes disruption of anticipatory (pre-target) EEG de-synchronization of alpha rhythms (about 8-12 Hz) in occipito-parietal cortex, mainly in the hemisphere contralateral to the locus of attention [62]. Moreover, the parietal area is associated to the function of interpretation and integration informations [28], being P4 derivation correspondent to the left side of the body [63]. In this way, the absence of requirement in interpretation and integration informations came from left side of the body resulted in increase of the alpha band absolute power, reflecting a state of relative neural inactivity on the analyzed region [42]. Complementarily, visual stimuli are secondarily processed in parietal areas [64], then an increased on alpha band absolute power in this region indicate that the stimuli did not required high levels of attention to be processed. In fact, the task can be considered easy to execute, once required only index movement (flexion and extension) as response to the visual stimulus who was presented.

Lastly, ours study find no significative difference in occipital region, as it was expected due no influence of immobilization to the function of the occipital cortical area which is more likely associated to process visual information [64]. In several clinic situations, the visual function have served as compensating sensory deficits, i.e. diabetic patient frequently uses vision to examine and then choses secure surroundings avoiding possible lesions on lower extremities affected by neuropathy [65]. Therefore, front to a situation of movement restriction, vision can assists the lack of afference linked to the immobilization and consequent no interaction of the segment in the environment. Our finding of no significative difference on alpha band absolute power at occiptal region between before and after hand immobilization indicates that this area kept exerting its function on similar way, despite immobilization. Therefore, our findings in occipital region demonstrate the no influence of hand immobilization on visual areas is a positive aspect because motor damage caused by immobilization can be, somewhat, minimized by visual attention [65]. Beyond that, the visual function diminished is associated with worst motor performance. A study involving force plate sway and gait velocity showed decrement in performance on subjects who had loss of vision [66].

\section{Conclusions}

The findings of our study allow concluding that there are cortical changes when a visual stimulus linked to a motor task is presented to the subject. Hand immobilization can causes limitation of sensory afferences and this lead to changes in cerebral cortex activation after 48 hours. Particularly, the study found changes on alpha band absolute power that denote a response to immobilization per 48 hours. At C4 derivation, lower values of alpha band absolute power indicate highest activation at this region as a compensatory strategy to execute the task after immobilization. The increased on alpha band absolute power at Fp1, Fp2, C3 and P4 indicate less activation of these regions after immobilization and may be due to 1) features of the task (easiness of execution); 2) learning of the task from the condition before immobilization; 3) habituation caused by repetitive stimuli; 4) memory lapse resulting of competition between visual stimulus and motor task; and 5) decrease of sensory-motor function generated by immobilization. In future studies be convenient analyze alpha band absolute power including rest as a third condition. Additionally, to before and after immobilization. Further studies are needed to better understand the complex processes involved in this type of task.

\section{Competing interests}

None of the authors have actual or potential conflict of interest including any financial, personal or other 
relationships with other people or organizations that could inappropriately influence, or be perceived to influence, our work.

\section{Acknowledgments}

OA-C is supported by CONACYT-BMBF 2013 (Grant 208132)

\section{Authors' contributions}

DM, ST, BV, PR, and OAC designed, conducted the experiment and drafted most of the manuscript. DM, VHVB, ST, MC, ASC, JSP and SM performed the experiment, the literature review and the drafting of the manuscript. All authors read and approved the final manuscript.

\section{References}

1. Kantak SS, Jones-Lush LM, Narayanan P, Judkins TN, Wittenberg GF. Rapid plasticity of motor corticospinal system with robotic reach training. Neurology 2012, 78 (3):182-188.

2. Langer $N$, Hänggi J, Müller NA, Simmen HP, Jäncke L. Effects of limb immobilization on brain plasticity. Neurocase, in press.

3. Fortuna M, Teixeira S, Machado S, Velasques B, Bittencourt J, Peressutti C, Budde $H$, Cagy M, Nardi $A E$, Piedade $R$, Ribeiro P, Arias-Carrion O. Cortical Reorganization after Hand Immobilization: The beta qEEG Spectral Coherence Evidences. PLoS One 2013, 8 (11): e79912.

4. McGeoch PD, Brang D, Huang M, Ramachandran VS. Primary somatosensory cortex hand representation dynamically modulated by motor output. Neurocase, in press.

5. Manaia F, Teixeira S, Velasques B, Bittencourt J, Salles JI, AriasCarrión O, Basile LF, Peressutti C, Carvalho MR, Cagy M, Piedade $R$, Ribeiro P, Machado S. Does immobilization of dependent hand promote adaptative changes in cerebral cortex? An analysis through qEEG asymmetry. Neuroscience Letters 2013, 538:20-25

6. Fabbri S, Strnad L, Caramazza A, Lingnau A. Overlapping representations for grip type and reach direction. Neurolmage 2014, 94:138-146.

7. Minc D, Machado S, Bastos V, Machado D, Cunha M, Cagy M, Budde H, Basile L, Piedade R, Ribeiro P. Gamma band oscillations under influence of bromazepam during a sensorimotor integration task: an EEG coherence study. Neuroscience Letters 2010, 469:145-149
8. Machado $D$, Bastos VH, Cunha $M$, Velasques $B$, Machado $S$, Basile L, Cagy M, Piedade R, Ribeiro P. Efectos del bromacepam en el desarrollo de una actividad sensoriomotora: un estudio electroencefalográfico. Rev Neurol 2009, 49(6):295-299.

9. Neuper C, Pfurtscheller G. Event-related dynamics of cortical rhythms: frequency-specific features and functional correlates. International Journal of Psychophysiology 2001, 43:41-58.

10. Cremades JG. The effects of imagery perspective as a function of skill level on alpha activity. International Journal of Psychophysiology 2002, 43:261-271.

11. Brignani D, Maioli C, Rossini PM, Miniussi C. Event-related power modulations of brain activity preceding visually guided saccades. Brain Research 2007, 1136:122-131.

12. Hale TS, Smalley SL, Hanada G, Macion J, McCracken JT, McGough JJ, Loo SK. Atypical alpha asymmetry in adults with ADHD. Neuropsychologia 2009, 47:2082-2088.

13. Varotto G, Fazio P, Sebastiano DR, Duran D, D'Incerti L, Parati E, Sattin D, Leonardi M, Franceschetti S, Panzica F. Altered resting state effective connectivity in long-standing vegetative state patients: An EEG study. Clinical Neurophysiology 2014, 125:6368.

14. Wheaton LA, Shibasaki H, Hallett M. Temporal activation pattern of parietal and premotor areas related to praxis movements. Clinical Neurophysiology 2005, 116:1201-1212.

15. Wheaton LA, Nolte G, Bohlhalter S, Fridman E, Hallet M. Synchronization of parietal and premotor areas during preparation and execution of praxis hand movements. Clinical Neurophysiology 2005, 116:1382-1390.

16. Wheaton LA, Fridman E, Bohlhalter S, Vorbach $S$, Hallet $M$. Left parietal activation related to planning, executing and suppressing praxis hand movements. Clinical Neurophysiology 2009, 120:980-986.

17. Babiloni $C$, Infarinato $F$, Marzano $N$, lacoboni $M$, Dassù $F$, Soricelli A, Rossini PM, Limatola C, DelPercio C. Intra-hemispheric functional coupling of alpha rhythms is related to golfer's performance: A coherence EEG study. International Journal of Psychophysiology 2011, 82:260-268.

18. Fortuna M, Teixeira S, Machado S, Velasques B, Bittencourt J, et al. Cortical reorganization after hand immobilization: the beta qeeg spectral coherence evidences. PLoS One 2013, 8(11): e79912.

19. Oldfield RC. The assessment and analysis of handedness: the Edinburgh inventory. Neuropsychologia 1971, 9:97-113.

20. Catanzariti JF, Guyot MA, Agnani O, Demaille S, Kolanowski E, Donze C. Eye-hand laterality and right thoracic idiopathic scoliosis. Eur Spine J 2014, in press.

21. Dixit A, Goyal A, Thawani R, Vaney N. Effect of caffeine on information processing: evidence from stroop task. Indian J Psychol Med. 2012, 34(3): 218-22. 
22. Knott $V$, Bisserbe JC, Shah D, Thompson A, Bowers $H$, Blais $C$, llivitsky $\mathrm{V}$. The moderating influence of nicotine and smoking on resting-state mood and EEG changes in remitted depressed patients during tryptophan depletion. Biol Psychol. 2013, 94(3):545-55

23. Martinovic J, Jones A, Christiansen P, Rose AK, Hogarth $L$, Field M. Electrophysiological responses to alcohol cues are not associated with pavlovian-to-instrumental transfer in social drinkers. PLoS One 2014, 14:9(4):e94605.

24. Daly I, Nicolaou N, Nasuto SJ, Warwick K. Automated Artifact Removal From the Electroencephalogram: A Comparative Study. Clinical EEG and Neuroscience 2013, 44(4):291-306.

25. Gross J. Analytical methods and experimental approaches for electrophysiological studies of brain oscillations. Journal of Neuroscience Methods 2014, 228:57-66.

26. Stewart AX, Nuthmann A, Sanguinetti G. Single-trial classification of EEG in a visual object task using ICA and machine learning. Journal of Neuroscience Methods 2014, 228:1-14.

27. Szurhaj $W$, Bourriez JL, Kahane $P$, Chauvel $P$, Mauguière $F$, et al. Intracerebral study of gamma rhythm reactivity in the sensorimotor cortex. Eur J Neurosci 2003, 21:1223-1235.

28. Teixeira S, Machado S, Velasques $B$, Sanfim A, Minc D, Peressutti C, Bittencourt J, Budde H, Cagy M, Anghinah R, Basile LF, Piedade R, Ribeiro P, Diniz C, Cartier C, Gongora M, Silva F, Manaia F, Silva J.G. Integrative parietal cortex processes: Neurological and psychiatric aspects. Journal of the Neurological Sciences 2014, 338:12-22.

29. Romei $V$, Brodbeck V, Michel C, Amedi A, Pascual-Leone A, Thut $\mathrm{G}$. Spontaneous fluctuations in posterior alpha-band EEG activity reflect variability in excitability of human visual areas. Cereb. Cortex 2008, 18:2010-2018.

30. Snyder AC, Foxe JJ. Anticipatory attentional suppression of visual features indexed by oscillatory alpha-band power increases: a high-density electrical mapping study. J. Neurosci. 2010, 30:4024-4032.

31. Jones SR, Kerr CE, Wan Q, Pritchett DL, Hamalainen M, Moore $\mathrm{Cl}$. Cued spatial attention drives functionally relevant modulation of the mu rhythm in primary somatosensory cortex. J. Neurosci. 2010, 30:13760-13765.

32. Haegens S, Handel BF, Jensen O. Top-down controlled alpha band activity in somatosensory areas determine behavioral performance in a discrimination task. J. Neurosci. 2011, 31:51975204

33. Domingues CA, Machado S, Cavaleiro EG, Furtado V, Cagy M, Ribeiro P, Piedade R. Alpha absolute power: motor learning of practical pistol shooting. Arq Neuropsiquiatr 2008, 66(2-B):336340.

34. Jiang Z, Zheng L. Inter- and intra-hemispheric EEG coherence in patients with mild cognitive impairment at rest and during working memory task. J Zhejiang Univ Sci B 2006, 7(5):357-364.
35. Van Albada SJ, Robinson PA. Transformation of arbitrary distributions to the normal distribution with application to EEG test-retest reliability. Journal of Neuroscience Methods 2007, 161: 205-211.

36. Dreher JC, Koechlin E, Tierney M, Grafman J. Damage to the fronto-polar cortex is associated with impaired multitasking. PLoS One 2008, 3(9):e3227.

37. Koechlin E, Basso G, Pietrini P, Panzer S, Grafman J. The role of the anterior prefrontal cortex in human cognition. Nature 1999, 399: 148-151.

38. Burgess PW, Quayle A, Frith CD. Brain regions involved in prospective memory as determined by positron emission tomography. Neuropsychologia 2001, 39:545-555.

39. Braver TS, Bongiolatti SR. The role of frontopolar cortex in subgoal processing during working memory. Neuroimage 2002, 15: $523-536$

40. Burgess PW, Scott SK, Frith CD. The role of the rostral frontal cortex (area 10) in prospective memory: a lateral versus medial dissociation. Neuropsychologia 2003, 41: 906-918.

41. Dias JC, Sajda P, Dmochowski JP, Parra LC. EEG precursors of detected and missed targets during free-viewing search. Journal of Vision 2013, 13(13):13, 1-19.

42. Kop WJ, Synowski SJ, Newell ME, Schmidt LA, Waldstein SR, Fox NA. Autonomic nervous system reactivity to positive and negative mood induction: The role of acute psychological responses and frontal electrocortical activity. Neuroscience 2013, 247:55-64.

43. Sauseng $P$, Klimesch $W$, Freunberger $R$, Pecherstorfer $T$, Hanslmayr S, Doppelmayr M. Relevance of EEG alpha and theta oscillations during task switching. Exp Brain Res 2006, 170(3):295-301.

44. Çevik MO. Habituation, sensitization, and Pavlovian conditioning. Frontiers in Integrative Neuroscience 2014, 8(13):1-6.

45. Steiner GZ, Barry RJ. The mechanism of dishabituation. Frontiers in Integrative Neuroscience 2014, 8(14):1-8.

46. Foxe JJ, Simpson GV. Biasing the brain's attentional set: effects of selective intersensory attentional deployments on subsequent sensory processing. Exp Brain Res 2005, 166:393-401.

47. Foxe JJ, Murphy JW, Sanctis P. Throwing out the rules: anticipatory alpha-band oscillatory attention mechanisms during task-set reconfigurations. European Journal of Neuroscience 2014, doi:10.1111/ejn.12577.

48. Van den Ber FE, Swinnen SP, Wenderoth N. Involvement of the primary motor cortex in controlling movements executed with the ipsilateral hand differs between left- and right-handers. J Cogn Neurosci 2011, 23(11):3456-69.

49. Langlet C, Bastide B, Canu MH. Hindlimb unloading affects cortical motor maps and decreases corticospinal excitability. Exp Neurol 2012, 237(1):211-7. 
50. Trinel D, Picquet F, Bastide B, Canu MH. Dendritic spine remodeling induced by hindlimb unloading in adult rat sensorimotor cortex. Behav Brain Res. 2013, 15(249):1-7.

51. Viaro R, Budri M, Parmiani P, Franchi G. Adaptive changes in the motor cortex during and after long-term forelimb immobilization in adult rats. J Physiol. 2014, in press.

52. Bittencourt J, Machado S, Teixeira S, Schlee G, Salles JI, Budde $H$, Basile LF, Narddi AE, Cagy M, Piedade R, Sack AT, Velasques B, Ribeiro P. Alpha-band power in the left frontal cortex discriminates the execution of fixed stimulus during saccadic eye movement. Neuroscience Letters 2012, 523:148-153.

53. Stark A, Meiner Z, Lefkovitz R, Levin N. Plasticity in cortical motor upper-limb representation following stroke and rehabilitation: two longitudinal multi-joint fMRI case-studies. Brain Topogr 2012, 25:205-219.

54. Yi W, Qiu S, Qi H, Zhang L, Wan B, Ming D. EEG feature comparison and classification of simple and compound limb motor imagery. J Neuroeng Rehabil 2013, 10:106.

55. Terada K, Umeoka S, Usui N, Baba K, Usui K, Fujitani S, Matsuda K, Tottori T, Nakamura F, Inoue Y. Uneven interhemispheric connections between left and right primary sensori-motor areas. Hum Brain Mapp 2012, 33(1):14-26.

56. Phillips KA, Hopkins WD. Tomography of the Chimpanzee corpus callosum. PLos One 2012, 7(2):e31941.

57. Simões EL, Bramati I, Rodrigues E, Franzoi A, Moll J, Lent R, TovarMoll F. Functional expansion of sensorimotor representation and structural reorganization of callosal connections in lower limb amputees. The Journal of Neuroscience 2012, 32(9):32113220 .

58. Vahdat S, Darainy M, Ostry DJ. Structure of plasticity in human sensory and motor networks due to perceptual learning. J Neurosci 2014, 34(7):2451-63.

59. Kamke MR, Ryan AE, Sale MV, Campbell ME, Riek S, Carroll TJ, Mattingley JB. Visual spatial attention has opposite effects on bidirectional plasticity in the human motor cortex. J Neurosci 2014, 34(4):1475-80

60. Chen X, Bin G, Daly I, Gao X. Event-related desynchronization (ERD) in the alpha band during a hand mental rotation task. Neurosci Lett 2013, 541:238-42.

61. Ruff CC, Blankenburg F, Bjoertomt O, Bestmann S, Weiskopf $\mathrm{N}$, Driver J. Hemispheric differences in frontal and parietal influences on human occipital cortex: direct confirmation with concurrent TMS-fMRI. J Cogn Neurosci 2009, 21(6):1146-61.

62. Capotosto P, Corbetta M, Romani GL, Babiloni C. Electrophysiological correlates of stimulus-driven reorienting deficits after interference with right parietal cortex during a spatial attention task: a TMS-EEG study. J Cogn Neurosci. 2012, 24(12):doi:10.1162/jocn_a_00287.

63. Jasper $\mathrm{HH}$. The ten twenty electrode system of the international federation. Electroenceph Clin Neurophysiol 1958, 10:371-375.
64. Innes-Brown H, Barutchu A, Crewther DP. Neural responses in parietal and occipital areas in response to visual events are modulated by prior multisensory stimuli. PLoS One 2012, 8(12):e84331.

65. Saurabh S, Sarkar S, Selvarai K, Kar SS, Kumar G, Roy G. Effectiveness of foot care education among people with type 2 diabetes in rural Puducherry, India. Indian J Endocrinol Metab. 2014, 18(1):106-110.

66. Damiano DL, Wingert JR, Stanley CJ, Curatalo L. Contribution of hip joint proprioception to static and dynamic balance in cerebral palsy: a case control study. Journal of NeuroEngineering and Rehabilitation 2013, 10:57.

\section{Comment on this article:}

\section{(f) (3) in \& $\mathbf{S}$ ?}

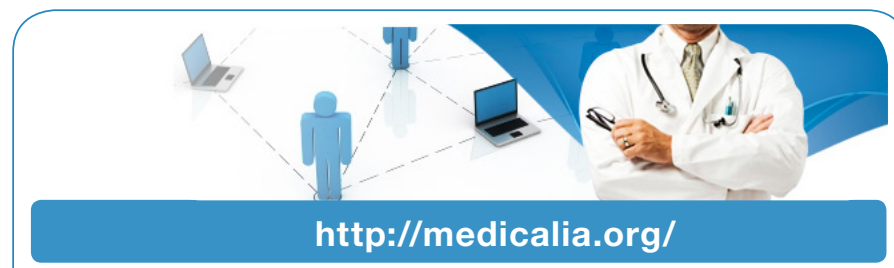

Where Doctors exchange clinical experiences, review their cases and share clinical knowledge. You can also access lots of medical publications for free. Join Now!

\section{Publish with iMedPub}

http://www.imed.pub

International Archives of Medicine is an open access journal publishing articles encompassing all aspects of medical science and clinical practice. IAM is considered a megajournal with independent sections on all areas of medicine. IAM is a really international journal with authors and board members from all around the world. The journal is widely indexed and classified Q1 in category Medicine. 